\title{
Utilizing Patient-specific 3D Printed Guides for Graft Reconstruction in Thoracoabdominal Aortic Repair
}

\section{Taehun Kim}

Department of Biomedical Engineering, Asan Medical Institute of Convergence Science and Technology, Asan Medical Center, University of Ulsan College of Medicine

\section{Dayeong Hong}

Department of Biomedical Engineering, Asan Medical Institute of Convergence Science and Technology, Asan Medical Center, University of Ulsan College of Medicine

Junhyeok Ock

Department of Convergence Medicine, Asan Medical Institute of Convergence Science and Technology, Asan Medical Center, University of Ulsan College of Medicine

\section{Sung Jun Park}

Department of Thoracic and Cardiovascular Surgery, Asan Medical Center, University of Ulsan College of Medicine

\section{Younju Rhee}

Department of Thoracic and Cardiovascular Surgery, Asan Medical Center, University of Ulsan College of Medicine

\section{Sangwook Lee}

ANYMEDI Inc.

\section{Guk Bae Kim}

ANYMEDI Inc.

\section{Dong Hyun Yang}

Department of Radiology, University of Ulsan College of Medicine, Asan Medical Center Joon Bum Kim

Department of Thoracic and Cardiovascular Surgery, Asan Medical Center, University of Ulsan College of Medicine

\section{Namkug Kim ( $\nabla$ namkugkim@gmail.com )}

Department of Convergence Medicine, Asan Medical Institute of Convergence Science and Technology, Asan Medical Center, University of Ulsan College of Medicine

\section{Research Article}

Keywords: 3D printing surgical guides, additive manufacturing, computer-aided design, aortic surgery, thoracoabdominal aortic aneurysm 
Posted Date: May 27th, 2021

DOI: https://doi.org/10.21203/rs.3.rs-556591/v1

License: (c) (i) This work is licensed under a Creative Commons Attribution 4.0 International License. Read Full License 


\section{Abstract}

In thoracoabdominal aortic aneurysm repair, repairing the visceral and segmental arteries is challenging. Although there is a pre-hand-sewn and multi-branched graft based on the conventional image-based technique, it has shortcomings in precisely positioning and directing the visceral and segmental arteries. Here, we introduce two new reconstruction techniques using patient-specific 3D-printed graft reconstruction guides: 1) model-based technique that presents the projected aortic graft, visualizing the main aortic body and its major branches and 2) guide-based technique in which the branching vessels in the visualization model are replaced by marking points identifiable by tactile sense. We demonstrate the effectiveness by evaluating conventional and new techniques based on accuracy, marking time requirement, reproducibility, and results of survey to surgeons on the perceived efficiency and efficacy. The graft reconstruction guides cover the segmentation, design, fabrication, post-processing, and clinical application of open surgical repair of thoracoabdominal aneurysm, and proved to be efficient for accurately reconstructing customized grafts.

\section{Background}

3D printing (3DP) technology is used in the medical field for patient-specified guides, simulators, surgical planning, education, and implants ${ }^{1-7}$. 3DP technologies, rapid prototyping and additive manufacturing, proceed by adding materials layer-by-layer until the object is completely built ${ }^{3,8}$. In addition to reducing the traditional processes, time, and cost, it also enables the fabrication of complex structures. In addition, using various materials, 3DP makes it possible to realise a virtual 3D model as a physical phantom, unlike existing technologies ${ }^{1,3,6,9-11}$

Aortic aneurysms are life-threatening disorders that increase the risks of aortic dissection or rupture with fatal outcomes ${ }^{12}$. Surgical or interventional therapies that anatomically replace the diseased aortic segments are the only treatments proven to prevent catastrophic aortic events. Within this disease entity, thoracoabdominal aortic aneurysm is the most extensive and challenging pathology; it requires the replacement of the diseased aorta including the downstream thoracic and abdominal segments, along with the reconstruction of the visceral arteries (celiac and superior mesenteric artery), bilateral renal arteries and several segmental arteries (intercostal and lumbar arteries) that supply blood to the spinal $\operatorname{cord}^{13}$. To perform this extensive surgical procedure, extracorporeal circulation, as well as selective perfusion to visceral and renal arteries are mandatory, both of which increase the invasiveness and level of surgical stress. Open surgical repair of thoracoabdominal aorta is well known as the most difficult and challenging surgical procedure, and operative outcomes remain reportedly poor, even by the world-leading expert groups, and the rates of surgical mortality, major stroke, and paraplegia are $9.5 \%, 11.6 \%$, and $13.9 \%$, respectively ${ }^{13}$.

Various efforts have been made to reduce such related risks in thoracoabdominal aorta surgery, including the use of the four-branched aortic graft for more efficient revascularizations of aortic branching arteries through the aid of commercially available products. In addition to this technique, the use of an eight- 
branched aortic graft-so called "octopod graft technique," has also been introduced to enable efficient revascularization of the spinal cord feeding arteries, which are known to be pivotal in preventing paraplegia ${ }^{14}$. The octopod technique involves constructing a pre-sewn multi-branched aortic graft before surgery through an image-based technique (IBT); it entails grafting branches for the intercostal and lumbar arteries (spinal cord suppliers) on commercially available four-branched grafts considering the anatomical relationship based on the preoperative computed tomography (CT) images because the anatomical location of these vessels significantly vary among patients ${ }^{15-18}$.

However, this conventional image-based approach has several shortcomings with respect to the precise positioning of the branching grafts because the accuracy depends on the constructor, usually, the operating surgeon. To overcome this limitation, 3DP-based reconstruction of the thoracoabdominal graft has been introduced to enable more accurate construction of the aorta and branching vessels ${ }^{4}$. As an extension of this approach, we developed two reconstruction techniques using patient-specific 3D printed graft reconstruction guides: 1) model-based technique (MBT), which uses a visualization model designed as a realistic-shaped graft model that contains the main aortic body and its branching vessels, making it possible to manually position the branching grafts on the artificial aortic graft and 2) guide-based technique (GBT) using the marking guides, wherein the branching vessels in the visualization model are substituted with slightly protruded marking points. We evaluated the accuracy and marking time efficiency of the three techniques (conventional IBT, MBT, and GBT) in terms of the proper positioning of the aortic branching vessels, using a designed graft model (DGM) as the gold standard.

\section{Results}

\section{Comparison of accuracy and marking time requirement of DGM and three techniques}

Table 1 presents the measurement of the diagonal line, height, and angle from the celiac artery to the segmental arteries for the DGM, whose design was validated by surgeons, conventional IBT, MBT, and GBT. According to the Bland-Altman analysis, the arithmetic mean and standard deviation (SD) of the difference between the DGM and conventional IBT were $-9.19 \pm 16.47 \mathrm{~mm}$ (limits of agreement (LoA): -41.47 to $23.08 \mathrm{~mm}$ ) for the diagonal line, $-9.25 \pm 16.84 \mathrm{~mm}$ (LoA: -42.26 to $23.76 \mathrm{~mm}$ ) for the height, and $-9.76^{\circ} \pm 10.05^{\circ}$ (LoA: $-29.47^{\circ}$ to $9.95^{\circ}$ ) for the angle (Fig. 1a-C), respectively; the difference between the DGM and MBT was $-1.67 \pm 8.11 \mathrm{~mm}$ (LoA: -17.56 to $14.21 \mathrm{~mm}$ ) for the diagonal line, $-1.50 \pm 8.60 \mathrm{~mm}$ (LoA: -18.35 to $15.36 \mathrm{~mm}$ ) for the height, and $-8.89^{\circ} \pm 10.93^{\circ}$ (LoA: $-30.31^{\circ}$ to $12.52^{\circ}$ ) for the angle (Fig. 1d-f), respectively. The arithmetic difference values for the diagonal line, height, and angle between the DGM and GBT were $-1.00 \pm 7.27 \mathrm{~mm}$ (LoA: -15.25 to $13.24 \mathrm{~mm}$ ), $1.79 \pm 4.93 \mathrm{~mm}$ (LoA: -7.86 to 11.45 $\mathrm{mm}$ ), and $-6.39^{\circ} \pm 10.29^{\circ}$ (LoA: $-26.55^{\circ}$ to $13.78^{\circ}$ ) (Fig. $1 \mathrm{~g}-\mathrm{i}$ ), respectively. There were statistically significant differences in all the measurements for the IBT, MBT, and GBT, except for the diagonal line of the GBT. The time requirements for graft marking for the three techniques are summarised in Table 1 . The 
MBT and GBT reduced the marking times by 17.6 and 15.5 min, respectively, compared with the conventional IBT.

Table 1

Measurements and time requirements of graft reconstruction using DGM, IBT, MBT, and GBT. DGM, designed graft model; IBT, image-based technique; GBT, guide-based technique.

\begin{tabular}{|c|c|c|c|c|c|c|c|}
\hline \multirow[t]{2}{*}{ Measurements } & \multirow{2}{*}{$\begin{array}{l}\text { DGM } \\
\begin{array}{l}\text { Mean } \\
\pm S D\end{array}\end{array}$} & \multicolumn{2}{|l|}{ IBT } & \multicolumn{2}{|l|}{ MBT } & \multicolumn{2}{|l|}{ GBT } \\
\hline & & $\begin{array}{l}\text { Mean } \\
\pm S D\end{array}$ & p & $\begin{array}{l}\text { Mean } \\
\pm S D\end{array}$ & p & $\begin{array}{l}\text { Mean } \pm \\
\text { SD }\end{array}$ & p \\
\hline $\begin{array}{l}\text { diagonal line } \\
(\mathrm{mm})\end{array}$ & $\begin{array}{l}82.19 \\
\pm 37.18\end{array}$ & $\begin{array}{l}91.38 \\
\pm 42.38\end{array}$ & $<001$ & $\begin{array}{l}83.87 \\
\pm 33.85\end{array}$ & 0.013 & $\begin{array}{l}83.19 \pm \\
33.27^{-}\end{array}$ & 0.053 \\
\hline Height (mm) & $\begin{array}{l}70.20 \\
\pm 43.99\end{array}$ & $\begin{array}{l}79.46 \\
\pm 50.42\end{array}$ & $\begin{array}{l}< \\
0.001\end{array}$ & $\begin{array}{l}71.70 \\
\pm 41.77\end{array}$ & 0.028 & $\begin{array}{l}68.41 \pm \\
41.91\end{array}$ & $\begin{array}{l}<.001 \\
0.001\end{array}$ \\
\hline Angle $\left(^{\circ}\right)$ & $\begin{array}{l}47.38 \\
\pm 18.67\end{array}$ & $\begin{array}{l}57.14 \\
\pm 23.21\end{array}$ & $<0.001$ & $\begin{array}{l}56.28 \\
\pm 22.31\end{array}$ & $<0.001$ & $\begin{array}{l}53.77 \pm \\
22.52\end{array}$ & $\begin{array}{l}< \\
0.001\end{array}$ \\
\hline Time & - & \multicolumn{2}{|c|}{$\begin{array}{l}18 \mathrm{~m} 26 \mathrm{~s} \pm 9 \mathrm{~m} \\
4 \mathrm{~s}\end{array}$} & \multicolumn{2}{|c|}{$48 s \pm 15 s$} & $\begin{array}{l}2 m 54 \\
s \pm 51 s\end{array}$ & $\begin{array}{l}< \\
0.001\end{array}$ \\
\hline
\end{tabular}

\section{Comparison of correlation among three techniques and observers}

The comparison results of the correlations coefficients of the DGM and the three techniques, IBT, MBT, and GBT, based on their respective diagonal length, height, and angle are shown in Table 2. All the correlation coefficients exhibited strong correlation with statistical significances. The intraclass correlation coefficients (ICC) indicated by the observations of three independent observers showed high levels of agreement with 0.986 for the conventional IBT and MBT, and 0.995 for GBT.

Table 2

Correlation coefficient between DGM and the three techniques for diagonal length, height, and angle. $r_{12}$, correlation coefficient for DGM and IBT; $r_{13}$, correlation coefficient for DGM and MBT; $\mathrm{r}_{14}$, correlation coefficient for DGM and GBT.

\begin{tabular}{|lllllllll|}
\hline \multirow{2}{*}{ Comparing correlation coefficient } & \multicolumn{2}{l}{ Diagonal line } & Height & \multicolumn{3}{c|}{ Angle } \\
\cline { 2 - 9 } & $\mathrm{r}_{12}$ & $\mathrm{p}$ & $\mathrm{r}_{13}$ & $\mathrm{p}$ & $\mathrm{r}_{14}$ & $\mathrm{p}$ \\
\hline DGM & 0.905 & $<0.001$ & 0.933 & $<0.001$ & 0.884 & $<0.001$ \\
& IBT & 0.959 & $<0.001$ & 0.970 & $<0.001$ & 0.858 & $<0.001$ \\
\hline MBT & 0.980 & $<0.001$ & 0.993 & $<0.001$ & 0.875 & $<0.001$ \\
\hline
\end{tabular}

\section{Survey}


The scores of the questionnaires are presented in Table 3. The categories in the questionnaire were designed to rate graft reconstruction guides with the conventional IBT, MBT, and GBT on understanding, usefulness, satisfaction, surgical outcome, and recommendability for use by other surgeons for graft reconstruction in thoracoabdominal aortic repair. The average score for the MBT and GBT with the graft reconstruction guides was $59.33 \pm 0.58$ out of 65 , whereas the conventional IBT had a relatively lower score of 20.67. Generally, the MBT and GBT scored higher than the conventional IBT with statistical significance.

\section{Table 3}

Score of the survey in relation to understanding, usefulness, satisfaction, surgical outcome, and recommendability for use in other applications for IBT without 3DP and MBT and GBT with 3DP.

\begin{tabular}{|llll|}
\hline Classification & IBT & MBT and GBT & P \\
\hline Understanding the anatomic structure & $1.92 \pm 0.79$ & $4.33 \pm 0.98$ & $<0.01$ \\
\hline Usefulness for graft reconstruction & $1.22 \pm 0.44$ & $4.88 \pm 0.33$ & $<0.01$ \\
\hline Satisfaction for graft reconstruction & $1.50 \pm 0.55$ & $4.83 \pm 0.41$ & $<0.05$ \\
\hline Surgical outcomes & $1.50 \pm 0.55$ & $4.67 \pm 0.52$ & $<0.05$ \\
\hline Recommendations for other applications & $1.67 \pm 0.52$ & $4.17 \pm 0.41$ & $<0.05$ \\
\hline
\end{tabular}

\section{Discussion}

We developed two reconstruction techniques using patient-specific 3D printing graft reconstruction guides: 1) MBT using the visualization model designed based on the realistic-shaped graft model that contains the main aortic body and its branching vessels, making it possible to manually position the branching grafts on the artificial aortic graft and 2) GBT using the marking guides, in which the branching vessels in the visualization model were replaced by slightly protruding marking points. The patientspecific 3D printed graft reconstruction guides may provide following benefits: 1) The marking time can be minimized by checking and marking the ideal position of the patient's segmental arteries in complex anatomy such as extremely tortuous spine and aorta. 2) With the improved accuracy and reproducibility of the aortic graft reconstruction, the techniques can be utilized easily even for those in the learning curves. 3) Owing to the improved procedural efficiency, the techniques may contribute to reducing the level of fatigue of operating surgeons during lengthy and exhausting surgery, thus increasing focus on important procedures.

Crawford extent II or III thoracoabdominal aortic aneurysm repair entails replacing the thoracoabdominal aorta and revascularising the visceral branches and intercostal or lumbar arteries simultaneously; therefore, it is regarded as the most extensive and challenging operation with high risks of surgery-related mortality and serious complications. The traditional Crawford's island technique-a single aortic patch containing all four openings of visceral branches anastomosed with graft by side-to-side fashion-was previously accepted as a standard technique because it reduced the procedural burden ${ }^{19}$. However, it is 
reportedly common for patch aneurysm to develop in the remnant aortic island, especially among those with connective tissue disorders such as Marfan syndrome $\mathrm{e}^{20-24}$. Although there are limited published reports on employing the pre-sewn multi-branched aortic graft in open repair of thoracoabdominal aortic aneurysm, its use is now a desirable option to prevent such aneurysm formation as observed in the conventional island-type aortic repair $22,24-26$. In addition, in cases where the ostia of the aortic branching arteries are displaced far away from each other, the use of a multi-branched graft may be the only way to effectively remove the diseased aorta.

Furthermore, paraplegia is a devastating complication of thoracoabdominal aortic aneurysm repair, particularly in extent II and III, and preventing it is a primary focus. Segmental arteries between T8 and L2 have been considered to play an important role in spinal cord protection and majority of institutes routinely incorporate large intercostal arteries into the surgery to restore spinal cord perfusion ${ }^{13,27}$. However, those arteries are mostly reimplanted as a single patch through side-by-side anastomosis, even when pre-sewn thoracoabdominal aortic aneurysm graft is used, which also increases the risk of future patch aneurysm, particularly in Marfan patients. To reconstruct a thoracoabdominal aorta using multibranched aortic graft personalized to reflect individual anatomical variation, we introduce the use of MBT of 3DP for the open surgical repair of thoracoabdominal aortic aneurysm with reasonable operative outcomes. Furthermore, recently, we incorporated the GBT into the MBT to complement it and improve the procedural efficiency and accuracy. The aim of this study is to quantitatively evaluate these techniques.

In addition to the complexity of this high-risk open repair, it is difficult to ensure that vital branch arteries are in the proper positions when constructing patient-specific grafts. To overcome these challenges, Park et al. developed the octopod technique, an eight-branched aortic graft for reconstruction based on the $\mathrm{IBT}^{14}$. However, because the graft was reconstructed by measuring the diagonal line, height, and angle based on $\mathrm{CT}$ images under anaesthetic induction in the operating room, it is difficult to understand the individual characteristics of patients in detail within a limited time, which affects the accuracy. For example, the second patient, who had Crawford extent II thoracoabdominal aortic aneurysm with severe scoliosis, had an extremely tortuous main aorta, affecting the visceral and segmental arteries, unlike the general anatomical structure ${ }^{4}$. Because the shape of the graft to be replaced should be connected to the aorta along the tortuous spine, the graft marked using the conventional IBT, calculated by slice thickness and number, may differ from the actual visceral arteries and segmental arteries positions. However, the MBT and GBT using graft reconstruction guides consisting of visualization model and marking guide efficiently reduce the graft marking time and solve anatomical hurdles. Using the three techniques, 135 mimic grafts (15 patients, three observers, and three techniques) ranging from aortic arch to iliac artery were evaluated in experiments. The grafts are a very realistic mimic of standard cylindrical model grafts they were used to bypass the expensive graft cost. The DGM, conventional IBT, MBT, and GBT were evaluated and compared based on graft marking accuracy and time requirements.

The errors of the experiments can be divided into marking error with medical images, 3DP errors, and human errors ${ }^{10,28,29}$. The marking errors of the conventional IBT were affected by the subjective 
measurement in CT images. The direction of the segmental arteries was measured from the centre points of the aorta and the segmental arteries, with the horizontal line. However, for the aneurysm patients, it manifested as elliptical, rather than circular, in the axial view, and the errors could be attributable to the incorrectness of the centre point of the aorta and the subjectivity of the centre point of the aorta and blood vessel (Supplementary Fig. S1) ${ }^{28,29}$. The differences of MBT and GBT arose from 3DP technologies errors, including machine and 3D printed model and guide errors. The machine error is triggered by environmental factors, such as temperature, humidity, and vibration, and the materials, resolution, and usage duration of the 3D printers. The 3D printed model and guide errors depend on the size, shape, printing direction, and printing angle of the guides and post-processing, including support removal, ultraviolet polymerization, and surface smoothing ${ }^{10,28}$. In addition, autoclave and steam sterilization with high temperatures can deform or distort the graft reconstruction guide ${ }^{30}$. To minimize such problems, we opted for ethylene oxide gas sterilization at a relatively low temperature. The three techniques were prone to human errors; errors also occurred when defined landmarks-diagonal line, height, and angle-are measured using a digital calliper and digital angle ruler in the experiments with the three observers. Even if only two landmarks were selected among the diagonal line, height, and angle, the third could be derived; however, the landmarks were measured directly without using a mathematical formula considering measurement error.

In the Bland-Altman analysis, the diagonal line, height, and angles in the conventional IBT at -45.96 to $33.83 \mathrm{~mm},-46.70$ to $33.34 \mathrm{~mm}$, and $-39.05^{\circ}$ to $13.05^{\circ}$, respectively, exhibited wider ranges of differences, compared to the MBT and GBT; the differences among the diagonal line and height tended to fall in the negative or positive region as the distance between the segmental artery and celiac artery increased, and the differences between the angles tended to be mostly distributed in the negative region from zero. Outliers tended to appear in the IBT as the distances from the celiac artery from the segmental arteries increased in the diagonal line and height, and occurred in patient with tortuous and swelling aneurysm aorta or scoliosis in the angle (Fig. 1a-c). In the MBT, the difference ranges for diagonal line, height, and angle were -21.92 to $20.39 \mathrm{~mm},-25.54$ to $18.73 \mathrm{~mm}$, and $-39.97^{\circ}$ to $23.04^{\circ}$, respectively, and -21.22 to $19.29 \mathrm{~mm},-12.56$ to $14.34 \mathrm{~mm}$, and $-40.61^{\circ}$ to $15.31^{\circ}$, respectively, in the GBT (Table 1 ). The diagonal line and height in the MBT and GBT were distributed in the region of the negative difference, as the measured distance was smaller, indicating a positive difference as the measured distance increased. The bias of the diagonal line and angles was greater in the GBT and the height was greater in the MBT (Fig. 1d-i). In the outliers of the MBT and GBT, the impact on the morphology of the aorta was greater than the distance of the segmental arteries. The MBT and GBT reduced the graft reconstruction time significantly, and the effect was proportional to the number of segmental arteries (Supplementary Fig. S2). For example, the graft reconstruction time for the first patient with the least number of segmental arteries was $10.5,0.5$, and 2.8 min with the conventional IBT, MBT, and GBT, respectively. For the fourteenth patient with 13 segmental arteries, graft reconstruction time was 36.5, 1.4, and 4.4 min with the IBT, MBT, and GBT, respectively. The average time requirement for the MBT and GBT reduced by 17.4 and 15.5 min, respectively, showing the effect reduced by more than six times compared to IBT. In 
addition, although, based on the marking time, the MBT was more efficient than the GBT, the GBT had superior accuracy. (Table 1 ).

The correlation coefficient $\left(r_{14}\right)$ for the diagonal line and height between the DGM and GBT was observed to be stronger than that of the IBT $\left(r_{12}\right)$ and MBT $\left(r_{13}\right)$, whereas a similar level of correlation was confirmed for the angles. Thus, it was implied that the GBT was the most similar to DGM (Table 2). In addition, the ICC was examined, to confirm the human error and the reproducibility of graft reconstruction among three observers in conventional IBT and new techniques. All the techniques showed high agreement, with 0.995 for the GBT and 0.986 for the IBT and MBT.

For the conventional IBT (without 3DP), and the MBT and GBT (with 3DP), questionnaires were administered to three surgeons with experience in the three techniques. The questionnaire had 26 questions classified into five categories: 1 ) level of contribution to understanding the anatomic structure, 2) usefulness for graft reconstruction, 3) satisfaction level for graft reconstruction, 4) benefits of 3DP technology to surgical outcomes, and 5) recommendability of 3DP for thoracoabdominal aorta surgery to other surgeons. For the contribution to understanding the anatomic structure, $58.3 \%$ of the answers indicated strong agreement, 25\%, agreement, and $8.33 \%$, neutral and disagreement for the MBT and GBT, whereas for the conventional IBT, $25 \%$ of the answers indicated neutral, $41.7 \%$, disagreement, and $33.3 \%$, strong disagreement. Under the usefulness for graft reconstruction, $88.9 \%$ of the answers indicated strong agreement and $11.11 \%$, agreement, in the MBT and GBT, whereas $22.2 \%$ indicated disagreement and $77.8 \%$, strong disagreement in the conventional IBT. Finally, for the MBT and GBT, satisfactoriness of graft reconstruction, surgical outcomes, and recommendability to other surgeons was $88.33 \%, 66.67 \%$, and $16.67 \%$ of strong agreement, and $16.67 \%, 33.33 \%$, and $83.33 \%$ of agreement, respectively, and, in the conventional IBT, $50 \%$ of disagreement and strong agreement were identified.

There are several limitations to this study. First, the procedures, from data acquisition to 3DP, lasted over a week, which made it difficult to apply the reconstruction techniques using patient-specific 3D printed graft reconstruction guides to open repair in patients with acute aortic rupture. Depending on the quality of the CT images, low-resolution images or slice thickness exceeding $3 \mathrm{~mm}$ made it difficult to identify the segmental arteries. In further studies, to reduce the graft reconstruction time, not only should the position of the segmental arteries on the graft be marked, functions, including the suturing and bonding process and the application for direct bioprinting process, should be developed.

\section{Methods}

The procedure for two types of graft guide techniques, MBT and GBT, are illustrated in Fig. 2. The visualization model and marking guides segmented based on CT images were designed, modelled, and exported into the standard tessellation language $(\mathrm{STL})$ format. Then, they were printed using ColorJet printing (CJP) and the stereolithography apparatus (SLA) technology, following which they underwent post-processing and ethylene oxide gas sterilization. Graft reconstruction was performed using the MBT and GBT for clinical application and the accuracy and time requirements were evaluated. 


\section{Dataset acquisition}

The imaging and clinical data of 15 patients diagnosed with Crawford extent II or III thoracoabdominal aortic aneurysm were used. These patients underwent open surgical aortic repair using 3DP in Asan Medical Center between Jan. 2017 and Feb. 2020. The segmental arteries to be reconstructed were selected from among those located between the T8 and L2 levels, and the main stem aortic graft reconstruction ranged from the left subclavian artery to infra-renal abdominal aorta or to the bilateral common iliac artery. The individual profiles of the subjects are detailed in Table 4. All the subjects underwent CT angiography (Siemens SOMATOM series, Siemens Healthcare) with 0.6-3 mm slice thickness and 70-120 kVp. The institutional review board for human investigations at Asan Medical Center approved this study with a waiver of informed consent from patients because of the use of retrospective clinical and imaging data. The data were de-identified, in accordance with the Health Insurance Portability and Accountability Act privacy rule. 
Table 4

Individual profiles of the subject patients including classification, level of segmental artery, and range of replacement. Rs, Right side; Ls, Left side; T, Thoracic; LSA, left subclavian artery; IMA, interior mesenteric artery; BIA, bilateral common iliac artery; RA, renal artery.

\begin{tabular}{|c|c|c|c|c|c|c|c|c|}
\hline \multirow{2}{*}{$\begin{array}{l}\text { Patients } \\
\text { No }\end{array}$} & \multirow[t]{2}{*}{ Age } & \multirow[t]{2}{*}{ Sex } & \multirow{2}{*}{$\begin{array}{l}\text { Operation } \\
\text { date }\end{array}$} & \multirow{2}{*}{$\begin{array}{l}\text { Classification } \\
\text { (Extent) }\end{array}$} & \multicolumn{3}{|c|}{ Level of segmental arteries } & \multirow{2}{*}{$\begin{array}{l}\text { Range of } \\
\text { graft } \\
\text { reconstruction }\end{array}$} \\
\hline & & & & & Side & Thoracic & Lumbar & \\
\hline \multirow[t]{2}{*}{1} & \multirow[t]{2}{*}{36} & \multirow[t]{2}{*}{ W } & \multirow{2}{*}{$\begin{array}{l}12 \text { Jan. } \\
2017\end{array}$} & \multirow[t]{2}{*}{ II } & Rs & 10 & \multirow[t]{2}{*}{-} & \multirow[t]{2}{*}{ LSA to IMA } \\
\hline & & & & & Ls & 9,10 & & \\
\hline \multirow[t]{2}{*}{2} & \multirow[t]{2}{*}{57} & \multirow[t]{2}{*}{ W } & \multirow{2}{*}{$\begin{array}{l}17 \text { Oct. } \\
2017\end{array}$} & \multirow[t]{2}{*}{ II } & Rs & 8,10 & \multirow[t]{2}{*}{-} & \multirow[t]{2}{*}{ LSA to BIA } \\
\hline & & & & & Ls & 12 & & \\
\hline \multirow[t]{2}{*}{3} & \multirow[t]{2}{*}{48} & \multirow[t]{2}{*}{ M } & \multirow{2}{*}{$\begin{array}{l}\text { 16-May- } \\
18\end{array}$} & \multirow[t]{2}{*}{ II } & Rs & \multirow{2}{*}{$\begin{array}{l}9,10,11 \\
12\end{array}$} & \multirow[t]{2}{*}{ - } & \multirow[t]{2}{*}{ LSA to RA } \\
\hline & & & & & Ls & & & \\
\hline \multirow[t]{2}{*}{4} & \multirow[t]{2}{*}{40} & M & 05 Jun. & I & Rs & 9,11 & - & LSA to IMA \\
\hline & & & & & Ls & 9,10 & & \\
\hline 5 & 37 & M & 19 Jun. & II & Rs & 11,12 & - & T-9 to BIA \\
\hline & & & & & Ls & 11 & & \\
\hline 6 & 46 & M & 19 Jul. & III & Rs & 11,12 & - & LSA to BIA \\
\hline & & & & & Ls & & & \\
\hline 7 & 33 & M & 06 Sep. & II & Rs & 8,9 & - & LSA to IMA \\
\hline & & & & & Ls & & & \\
\hline 8 & 22 & $M$ & 04 Jul. & II & Rs & 10,11 & - & LSA to RA \\
\hline & & & & & Ls & & & \\
\hline 9 & 65 & $M$ & 27 Jun. & III & Rs & 10 & - & T- 10 to BIA \\
\hline & & & & & Ls & 11 & & \\
\hline 10 & 51 & W & 07 Nov. & III & Rs & 10,11 & 1 & LSA to BIA \\
\hline & & & & & Ls & 10 & 1 & \\
\hline 11 & 69 & M & 14 Nov. & II & Rs & $8,11,12$ & - & LSA to BIA \\
\hline & & & & & Ls & 11 & & \\
\hline 12 & 57 & $M$ & 22 Nov. & IV & Rs & 10,12 & - & LSA to CA \\
\hline & & & & & Ls & $9,10,12$ & & \\
\hline 13 & 22 & $M$ & $\begin{array}{l}19 \text { Dec. } \\
2019\end{array}$ & II & Rs & $9,10,12$ & 1,4 & T- 9 to BIA \\
\hline
\end{tabular}




\begin{tabular}{|c|c|c|c|c|c|c|c|c|}
\hline & & & & & Ls & 11,12 & 1 & \\
\hline \multirow[t]{2}{*}{14} & \multirow[t]{2}{*}{38} & \multirow[t]{2}{*}{$M$} & \multirow[t]{2}{*}{$\begin{array}{l}09 \text { Jan. } \\
2020\end{array}$} & \multirow[t]{2}{*}{ III } & Rs & $\begin{array}{l}7,8,10 \\
11,12\end{array}$ & 1,3 & \multirow[t]{2}{*}{$\mathrm{T}-7$ to BIA } \\
\hline & & & & & Ls & $\begin{array}{l}7,8,9 \\
10,11 \\
12\end{array}$ & $\begin{array}{l}7,8,9, \\
10,11, \\
12\end{array}$ & \\
\hline \multirow[t]{2}{*}{15} & \multirow[t]{2}{*}{40} & \multirow[t]{2}{*}{$M$} & \multirow{2}{*}{$\begin{array}{l}23 \text { Jan. } \\
2020\end{array}$} & \multirow[t]{2}{*}{ II } & Rs & $8,10,12$ & $2,3,4$ & \multirow[t]{2}{*}{ LSA to BIA } \\
\hline & & & & & Ls & 8,11 & 3,4 & \\
\hline
\end{tabular}

\section{Conventional image-based technique.}

The procedure of the conventional IBT proposed by Park et al. ${ }^{14}$ is as follows: 1) In the axial view of the CT images, the position of the visceral and segmental (intercostal and lumbar) arteries are identified. 2) To evaluate the angle between the visceral and segmental arteries, a horizontal line and a virtual diverging line of the branch from the central spot of aorta are drawn on each axial slices of a CT image at the visceral and segmental levels, and the angles between both lines are measured at each level. 3) Based on the visceral artery, the height of the segmental artery is determined and the corresponding point is marked with measured angle on the main aortic graft. 4) These processes are repeated as many times as the number of segmental arteries to be reconstructed.

\section{Development of visualization model and marking guide}

In manually segmenting the native aorta and spine, the major aortic branching vessels-the celiac artery, superior mesenteric artery, renal artery, bilateral common iliac artery, segmental arteries - using Mimics Medical 17 and 3-matic 9.0 software (Materialise Inc, Belgium) (Fig. 3a) were included. After 3D rendering based on the segmentation, the centreline of the native aorta was extracted through 3D computer-aided design (CAD) modelling and the squiggly centreline was modified to reflect the spine shape (Fig. 3b). The virtual model of the aorta was designed in the diameter of the prospective aortic graft to be implanted (24-30 mm); each of the virtual side-branches were united in $8 \mathrm{~mm}$ diameter as a single model. To achieve more efficient and accurate construction of the aortic graft, the graft reconstruction model and guide, visualization model, and marking guide, were developed: 1) The visualization model was designed as a realistic-shaped graft model containing the main aortic body and its branching vessels, to enable manual positioning of the branching grafts on the artificial aortic graft (Fig. 3c-left); 2) In the marking guide, the branching vessels in the visualization model were replaced by slightly protruding marking points $(2 \mathrm{~mm}$ ), to ensure that their positions were designed such as to be identifiable by tactile sense when wrapped by the aortic graft, instead of depending solely on visual mimicking (Fig. 3c-right). The DGM was validated by operating surgeons, following which it was exported as STL files. 


\section{D printing technology}

The visualization model was fabricated using a ColorJet printer (CJP) with VisiJet PXL Core powder, VisiJet PXL clear binder, and colour bonding materials (Fig. 3d-left). The marking guide was printed using a stereolithography apparatus (SLA) with Dental LT resin, a material capable of temporary mucosal contact (Fig. 3d-right). To fabricate the graft reconstruction guides, the layer thickness, field of view and $X-Y$ plane resolution for the CJP were $0.10,254 \times 381 \times 203$ and $300 \times 450 \mathrm{DPI}$, respectively, and 0.10 , $145 \times 145 \times 145,0.15 \mathrm{~mm}$, respectively, for the SLA. The X-Y resolution of SLA was not precisely defined; therefore, Formlabs Inc. experimented the specimens to find the $X-Y$ resolution ${ }^{31}$. In addition, the spot size and power of laser were $140 \mathrm{um}$ and $250 \mathrm{~mW}$.

\section{Graft reconstruction method using MBT and GBT}

In a real operative setting, the grafts were pre-sewn by the operating surgeon with the patient during anaesthetic induction using either the MBT or GBT, and were used to surgically replace the diseased thoracoabdominal aorta (Fig. 3e). The postoperative configuration of the replaced aorta was confirmed by CT images (Fig. 3f). The experimental processes for patient-specific graft reconstruction using MBT and GBT are shown in Fig. 4. Under the supervision of the visualization model in the MBT, the shape of the main graft body was imitated (Fig. $4 \mathrm{a}$ ) and the locations of the segmental vessels were indicated on the main graft body, with the point of the celiac artery origin as reference (Fig. 4b). In the GBT, the marking guide was inserted into the aortic graft and aligned with reference to the origin of the visceral arteries (Fig. 4c). Then, the positions of the segmental arteries were determined by the tactile sense through the protruding markers and marked on the graft surface (Fig. 4d). Both in the MBT and GBT, the segmental grafts were attached to the marked points by running sutures (Fig. 4e).

\section{Survey}

To assess the convenience availed by the proposed techniques, three practicing cardiothoracic surgeons with experience in graft reconstruction with the three techniques, IBT, MBT and GBT, were surveyed. The survey questionnaire was constructed in-house by curating aids to anatomical understanding, usefulness, satisfaction, surgical outcome, and reccomendability from clinical standpoints. The surveyees had 3 to 10 years of experience as certified cardiothoracic surgeons.

The questionnaire items were categorised as follows: 1) the level of contribution to understanding the anatomic structure (four questions); 2) usefulness for graft reconstruction (three questions); 3) satisfaction level for graft reconstruction (two questions); and 4) benefits of the use of 3DP technologies to surgical outcomes (two questions); and 5) recommendability of 3DP for thoracoabdominal aorta surgery to other surgeons (two questions). The scores were recorded as $1=$ Strong disagreement, 2 = Disagreement, 3 = Neutral, 4 = Agreement, and 5 = Strong agreement. 


\section{Evaluation and Statistical analysis}

The conventional IBT, MBT, and GBT were measured, analysed, and compared with the DGM based on the graft reconstruction accuracy and time requirement. Experiments of three techniques were performed by fabricated mimic aortic grafts. In the accuracy evaluation, the positions of the segmental arteries were indicated on the graft using the IBT, MBT and GBT (Fig. 5a), and the graft was unfolded using scissors (Fig. 5b). Then, the diagonal line, height, and angle between the designated celiac artery and the marked segmental arteries were measured (Fig. 5c).

The Bland-Altman analysis was used to compare the differences in the diagonal line, height, and angles of the DGM and the three techniques, IBT, MBT and GBT, and to identify the LoA, bias, and outliers ${ }^{32,33}$. The inter-group differences in the measurements and marking time were evaluated using the paired t-test. The correlation coefficients ( $r$ ) for the DGM and the three techniques were observed, and based on the correlation analyses of the same sample size by an independent group, it was determined that the two correlations were statistically significant. An ICC analysis was performed on the IBT, MBT and GBT experiments, with three observers to confirm the correlations among them. In addition, the survey results on the IBT and the MBT or GBT were analysed using the Wilcoxon signed rank test. All the statistical analysis was conducted using statistics software (SPSS 25.00, IBM; and Medcalc 19.6.4 free trial, Medcalc Software).

\section{Conclusion}

In this study, two types of grafts were developed for patient-specific graft reconstruction. Both methods provided a useful individualised approach to the anatomically challenging open repair. The MBT and GBT effectively reduced the graft reconstruction time and improved the accuracy, compared to the IBT.

\section{Declarations}

\section{Acknowledgements}

Minkyeong Kim who is affiliated to department of Convergence Medicine, Asan Medical Center assisted with the drawings in Figure 4 and 5 and You Jung Ok, a member of department of Thoracic and Cardiovascular Surgery, Asan Medical Center carried out the survey.

\section{Author Contributions}

T.K. and J. -B.K. wrote the texts of the main manuscript, figures, and tables. T.K., S.L., and G. -B. K. developed the graft reconstruction guides. T.K., D.H., and J.O. collaborated on the data measurement. D.H.Y. Y.R., S.-J.P., and J.-B.K. lent their clinical expertise. This study and the revision of the manuscript were supervised by N.K. and J.-B. K., based on their knowledge and experiences. All the authors reviewed the manuscript. 


\section{Funding}

This study was supported by a grant of the Korea Health Technology R\&D Project through the Korea Health Industry Development Institute (KHIDI), funded by the Ministry of Health \& Welfare, Republic of Korea (HI18C2383).

\section{Competing Interests}

The authors declare no competing interests.

\section{References}

1. Gross, B. C., Erkal, J. L., Lockwood, S. Y., Chen, C. \& Spence, D. M. Evaluation of 3D printing and its potential impact on biotechnology and the chemical sciences. ACS Publications. 86, 3240-3253 (2014).

2. Hong, D. et al. Usefulness of a 3D-printed thyroid cancer phantom for clinician to patient communication. World J. Surg. 44, 788-794 (2020).

3. Kim, G. B. et al. Three-dimensional printing: basic principles and applications in medicine and radiology. Korean J. Radiol. 17, 182-197 (2016).

4. Kim, W. K. et al. 3D-printing-based open repair of extensive thoracoabdominal aorta in severe scoliosis. Semin. Thorac. Cardiovasc. Surg. 31, 61-63 (2019).

5. Michalski, M. H. \& Ross, J. S. The shape of things to come: 3D printing in medicine. JAMA. 312, 2213-2214 (2014).

6. Murphy, S. V. \& Atala A. 3D bioprinting of tissues and organs. Nat. Biotechnol. 32, 773 (2014).

7. Rengier, F. et al. 3D printing based on imaging data: review of medical applications. Int. J. Comput. Assist. Radiol. Surg. 5, 335-341 (2010).

8. Mitsouras, D. et al. Medical 3D printing for the radiologist. Radiographics. 35, 1965-1988 (2015).

9. Hong, D. et al. Development of a personalized and realistic educational thyroid cancer phantom based on CT images: An evaluation of accuracy between three different 3D printers. Comput. Biol. Med. 113, 103393 (2019).

10. Kim, T. et al. Accuracy of a simplified 3D-printed implant surgical guide. The Journal of prosthetic dentistry. 124, 195-201 (2020).

11. McGurk, M., Amis, A., Potamianos, P. \& Goodger, N. Rapid prototyping techniques for anatomical modelling in medicine. Ann. R. Coll. Surg. Engl. 79, 169 (1997).

12. Crawford, E. S. \& DeNatale, R. W. Thoracoabdominal aortic aneurysm: observations regarding the natural course of the disease. J. Vasc. Surg. 3, 578-582 (1986).

13. Coselli, J. S. et al. Outcomes of 3309 thoracoabdominal aortic aneurysm repairs. The Journal of thoracic and cardiovascular surgery. 151, 1323-1338 (2016). 
14. Park, S. J. \& Kim, J. B. An eight-branched aortic graft for reconstruction of visceral and intercostal arteries during extent II thoraco-abdominal aortic surgery. Eur. J. Cardiothorac. Surg. 53, 1282-1283 (2017).

15. Estrera, A. L. The artery of Adamkiewicz: More interesting than practical? The Journal of thoracic and cardiovascular surgery. 151, 129-130 (2016).

16. Katsargyris, A. et al. Spinal cord ischemia after endovascular repair of thoracoabdominal aortic aneurysms with fenestrated and branched stent grafts. J. Vasc. Surg. 62, 1450-1456 (2015).

17. Tanaka, H. et al. The impact of preoperative identification of the Adamkiewicz artery on descending and thoracoabdominal aortic repair. The Journal of thoracic and cardiovascular surgery. 151, 122128 (2016).

18. Etz, C. D. et al. Thoracic and thoracoabdominal aneurysm repair: is reimplantation of spinal cord arteries a waste of time? The Annals of thoracic surgery. 82, 1670-1677 (2006).

19. Crawford, E. S. Thoraco-abdominal and abdominal aortic aneurysms involving renal, superior mesenteric, celiac arteries. Ann. Surg. 179, 763 (1974).

20. Dardik, A., Perler, B. A., Roseborough, G. S. \& Williams, G. M. Aneurysmal expansion of the visceral patch after thoracoabdominal aortic replacement: an argument for limiting patch size? J. Vasc. Surg. 34, 405-410 (2001).

21. Tshomba, Y., Melissano, G., Civilini, E., Setacci, F. \& Chiesa, R. Fate of the visceral aortic patch after thoracoabdominal aortic repair. Eur. J. Vasc. Endovasc. Surg. 29, 383-389 (2005).

22. Kokotsakis, J. et al. Thoracoabdominal aneurysm repair using a four-branched thoracoabdominal graft: a case series. Cases journal. 2, 1-4 (2009).

23. Kulik, A., Allen, B. T. \& Kouchoukos, N. T. Incidence and management of intercostal patch aneurysms after repair of thoracoabdominal aortic aneurysms. The Journal of thoracic and cardiovascular surgery. 138, 352-358 (2009).

24. De Rango, P. et al. Operative outcomes using a side-branched thoracoabdominal aortic graft (STAG) for thoraco-abdominal aortic repair. Eur. J. Vasc. Endovasc. Surg. 41, 41-47 (2011).

25. Kim, I., LeMaire, S. A., Weldon, S. A. \& Coselli, J. S. Thoracoabdominal aortic aneurysm repair with a branched graft. Annals of cardiothoracic surgery. 1, 381 (2012).

26. Kouchoukos, N. T., Kulik, A. \& Castner, C. Branch graft patency after open repair of thoracoabdominal aortic aneurysms. The Journal of thoracic and cardiovascular surgery. 153, S14-S19 (2017).

27. Estrera, A. L. et al. A quarter century of organ protection in open thoracoabdominal repair. Ann. Surg. 262, 660-668 (2015).

28. Choi, J. Y. et al. Analysis of errors in medical rapid prototyping models. Int. J. Oral Maxillofac. Surg. 31, 23-32 (2002).

29. Koleilat, I. et al. Interobserver variability in physician-modified endograft planning by comparison with a three-dimensional printed aortic model. J. Vasc. Surg. 64, 1789-1796 (2016). 
30. Chen, J. V., Tanaka, K. S., Dang, A. B. \& Dang, A. Identifying a commercially-available 3D printing process that minimizes model distortion after annealing and autoclaving and the effect of steam sterilization on mechanical strength. 3D Printing in Medicine. 6, 1-10 (2020).

31. What Does Resolution Mean in 3D Printing? https://formlabs.com/blog/3d-printer-resolutionmeaning/.

32. Bland, J. M. \& Altman, D. Statistical methods for assessing agreement between two methods of clinical measurement. The lancet. 327, 307-310 (1986).

33. Hazeveld, A., Slater, J. J. H. \& Ren, Y. Accuracy and reproducibility of dental replica models reconstructed by different rapid prototyping techniques. Am. J. Orthod. Dentofacial Orthop. 145, 108-115 (2014).

\section{Figures}
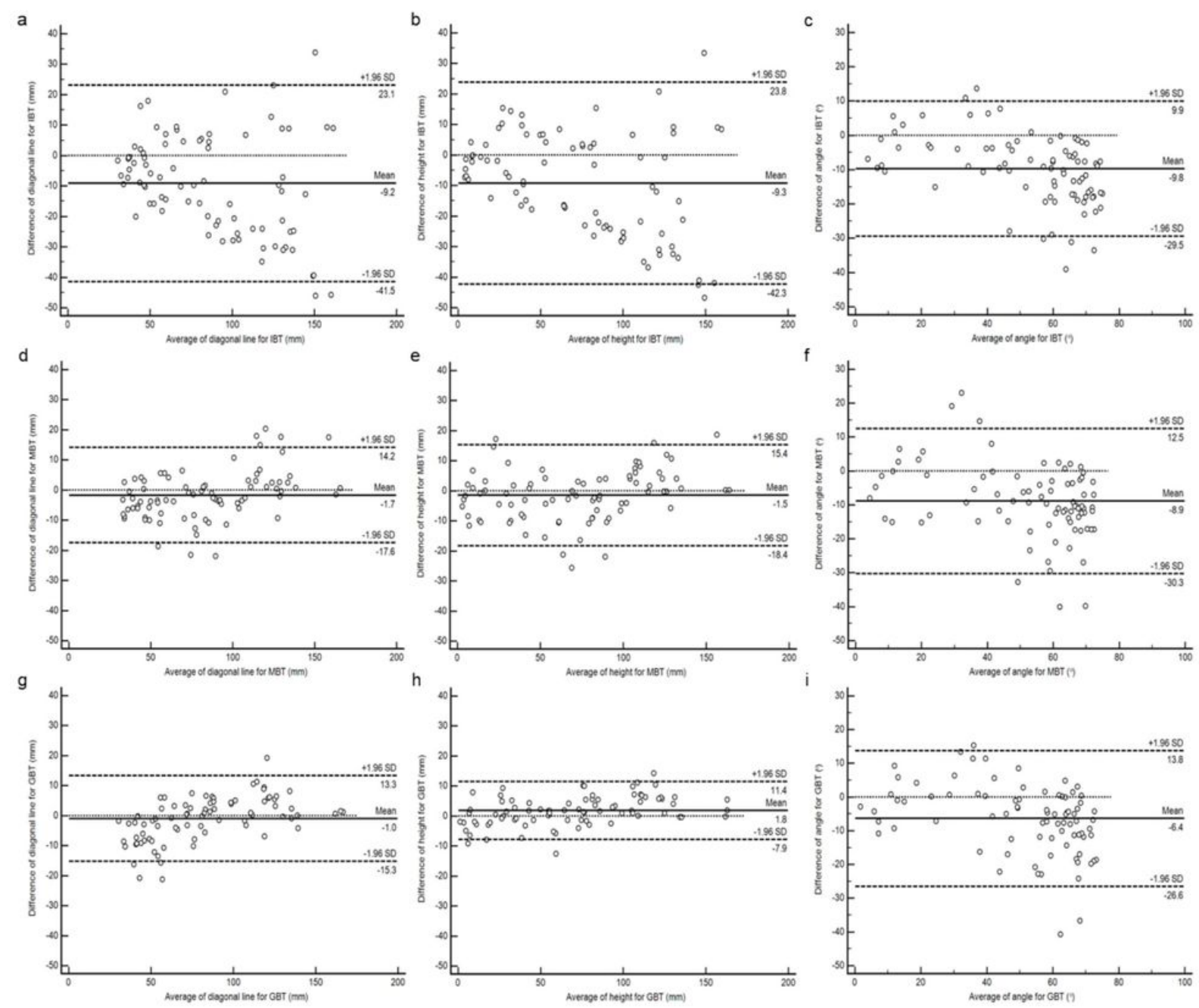

Figure 1 
Bland-Altman plot indicating the distribution of the differences between DGM and IBT, divided by a. diagonal line, b. height and c. angle; between the DGM and MBT, divided by d. diagonal line, e. height, $f$. angle; and between the DGM and GBT, divided by g. diagonal line, h. height, and i. angle.

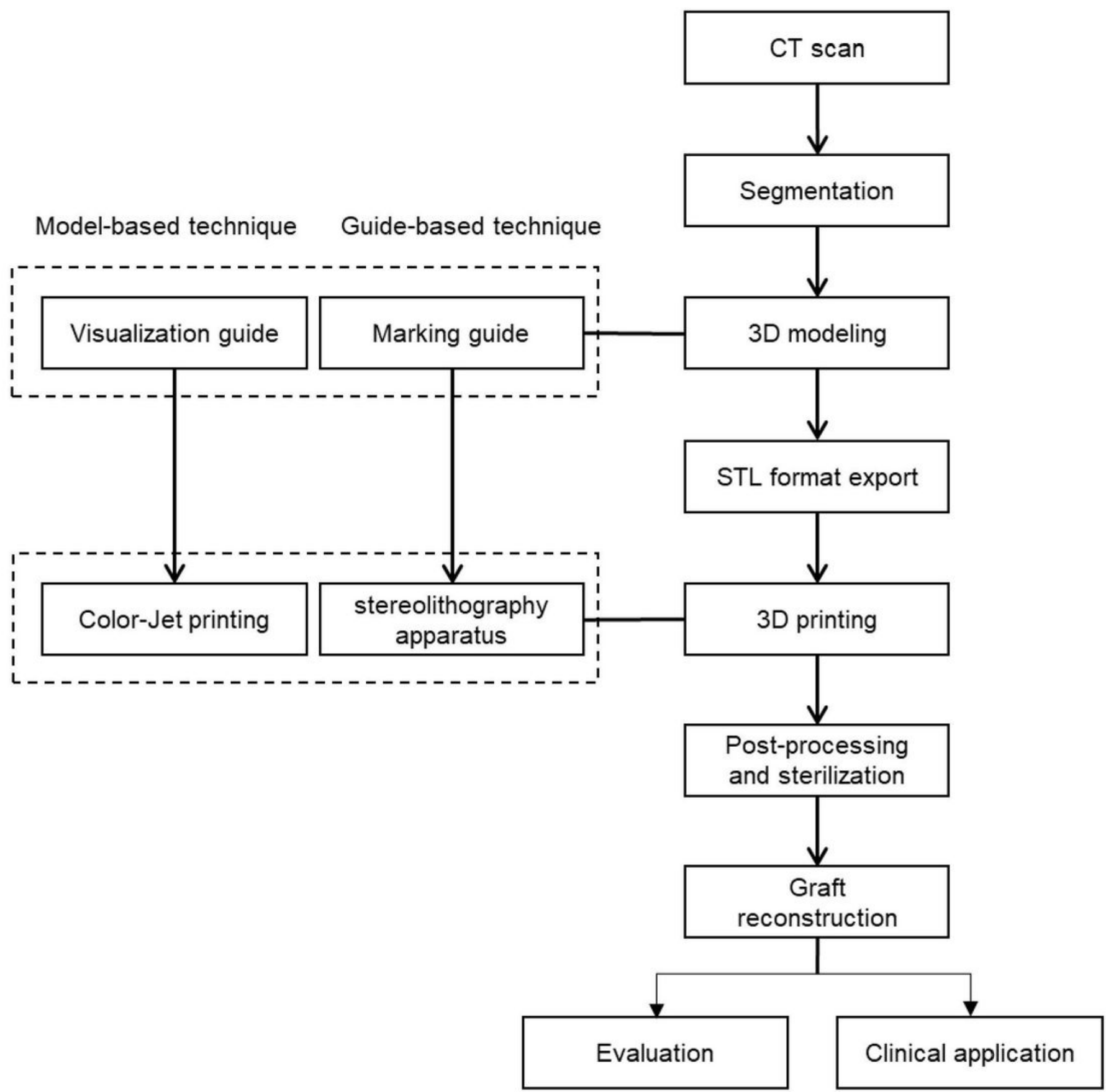

Figure 2

Overall process of two types of graft guide with MBT and GBT. 

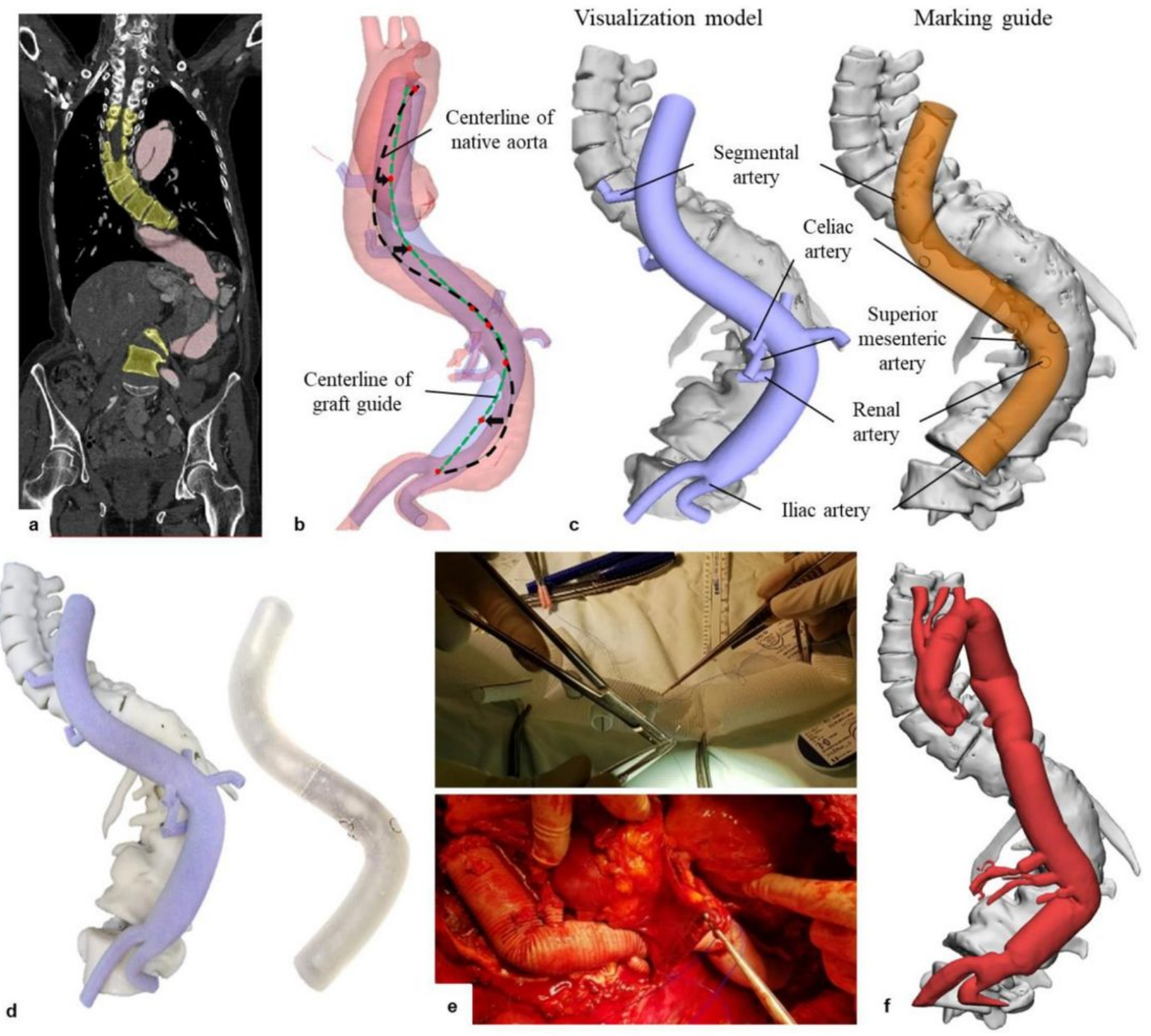

\section{Figure 3}

Two types of patient-specific graft reconstruction guide application for open repair of thoracoabdominal aortic aneurysm. a. CT angiography images and segmentation of diseased aorta and spine; $b$. Modelling the centreline of graft based on that of a native aorta; c. 3D modelling of the visualization model and marking guide; d. 3D-printed visualization model and marking guide; e. Graft reconstruction and clinical application in operating room; f. Postoperative graft. 

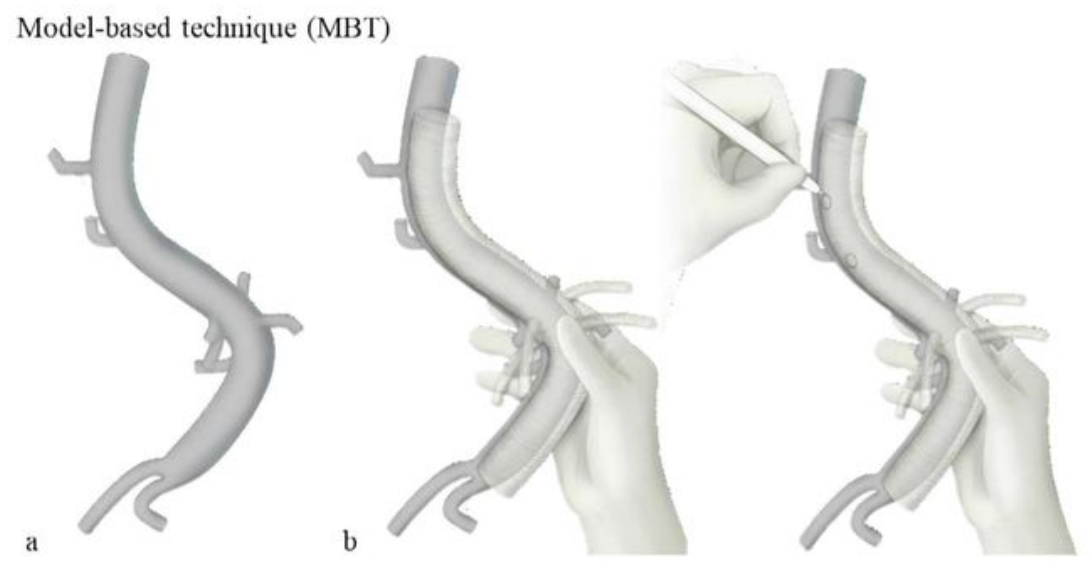

Guide-based technique (GBT)

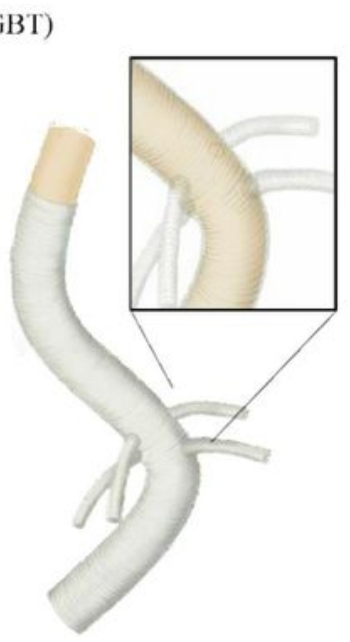

c
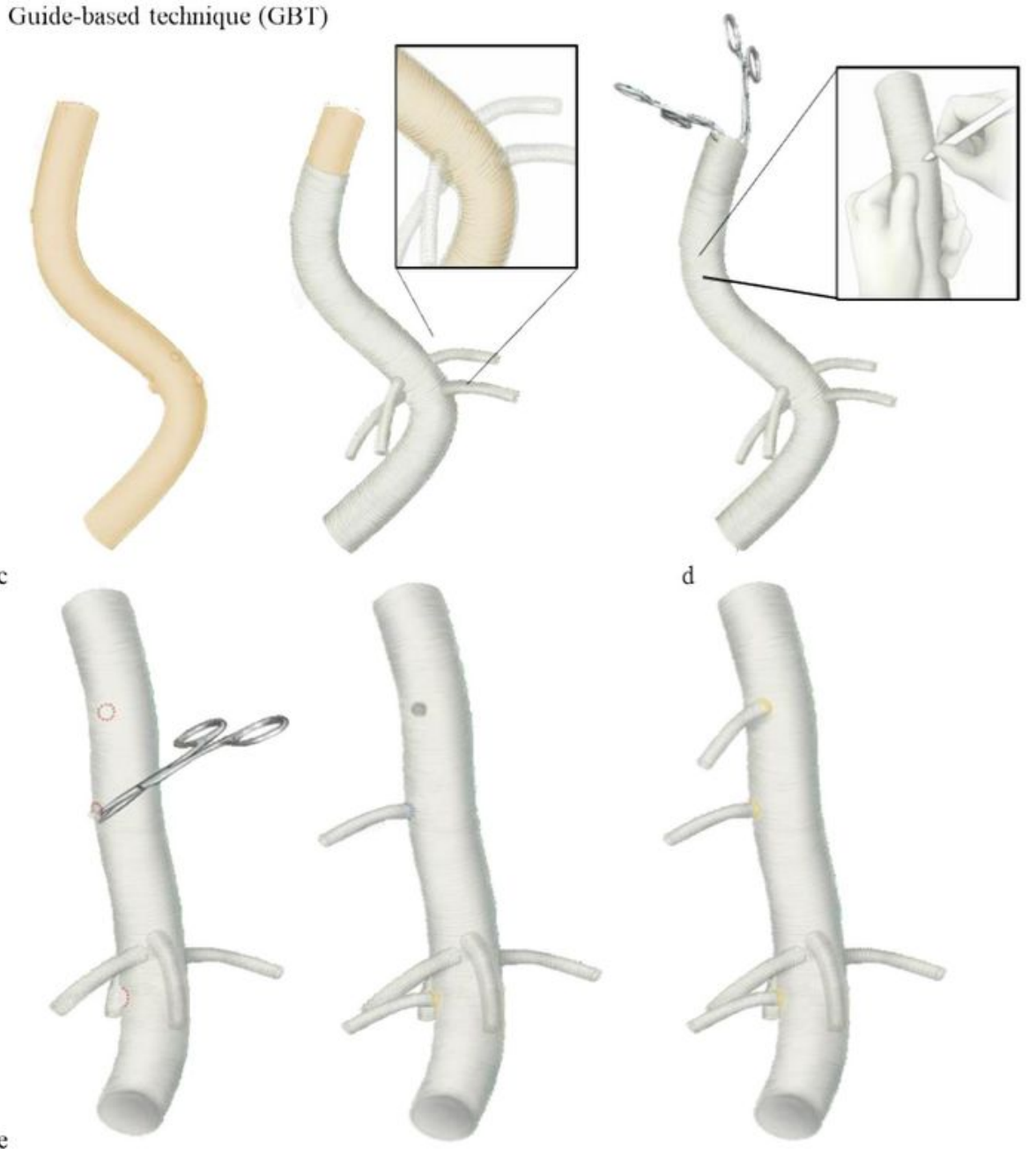

\section{Figure 4}

Patient-specific graft reconstruction process with MBT and GBT in operating room. 

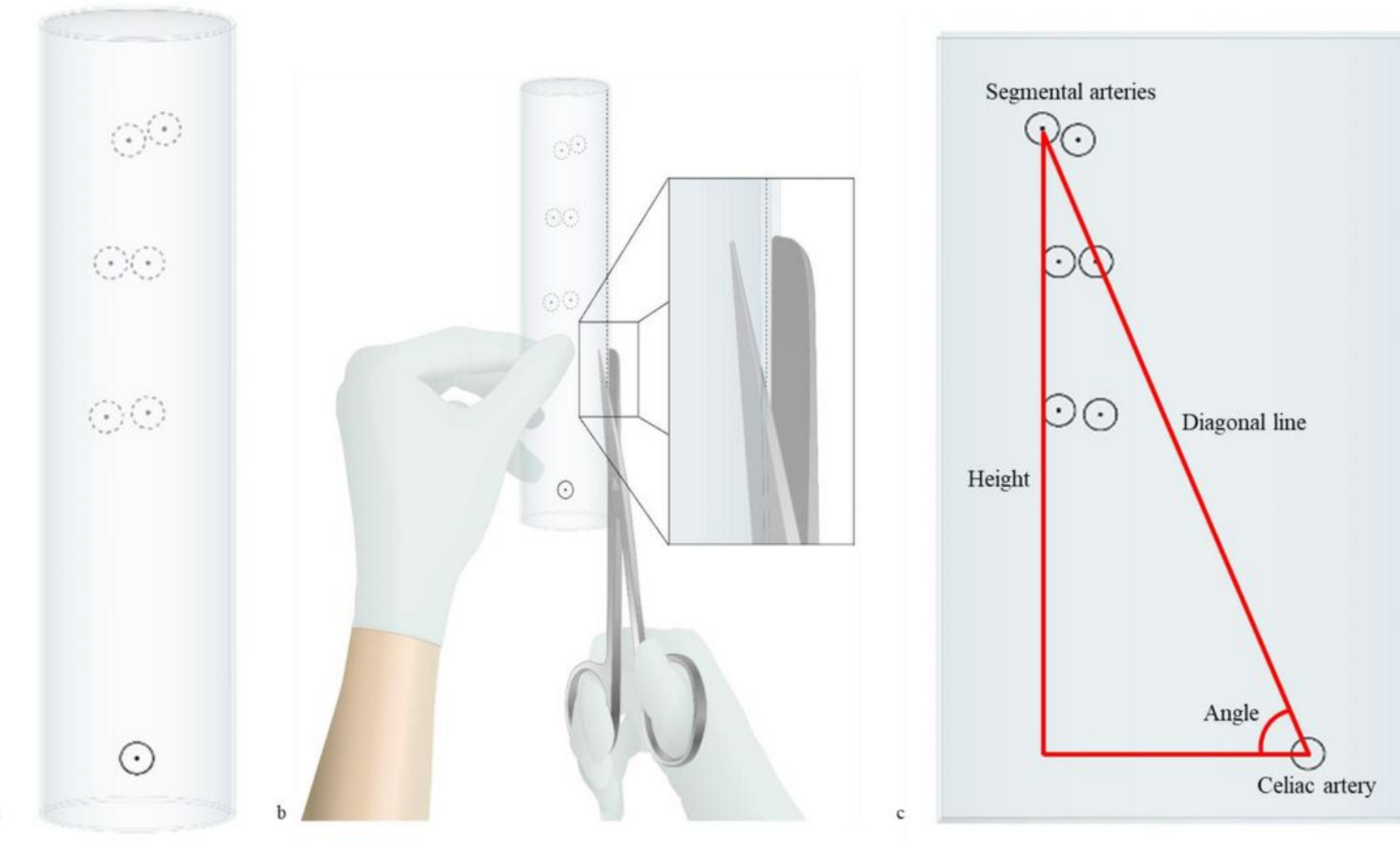

\section{Figure 5}

Guide to evaluating the accuracy of IBT and GBT; a. Locating celiac artery and segmental arteries marked on graft. b, Spreading the graft using scissors. c, Measuring the length, height, and angle between celiac artery and segmental arteries.

\section{Supplementary Files}

This is a list of supplementary files associated with this preprint. Click to download.

- SupplementaryFigureS1.pdf

- SupplementaryFigureS2.pdf 